\title{
Some continuum physics results from the lattice V-A correlator
}

\author{
P.A. Boyle, ${ }^{a}$, L. Del Debbio, ${ }^{a}$ N. Garron, ${ }^{b}$, R.J. Hudspith, ${ }^{a}$, E. Kerrane ${ }^{c}$, K. Maltman* \\ ,$^{d, e}$ and J.M. Zanotti ${ }^{e}$ \\ ${ }^{a}$ Physics and Astronomy, University of Edinburgh, Edinburgh EH9 3JZ, UK \\ ${ }^{b}$ School of Mathematics, Trinity College, Dublin 2, Ireland \\ ${ }^{c}$ Instituto de Fìsica Tèorica UAM/CSIC, Universidad Autònoma de Madrid, Cantoblanco \\ E-28049 Madrid, Spain \\ ${ }^{d}$ Mathematics and Statistics, York University, Toronto M3J 1P3 Canada \\ ${ }^{e}$ CSSM, University of Adelaide, Adelaide 5005 Australia \\ E-mail: paboyle@ph.ed.ac.uk, ldeldebb@ph.ed.ac.uk, \\ ngarron@maths.tcd. ie, s0968574 @sms.ed.ac.uk, \\ eoin.kerrane@gmail.com, kmaltman@yorku.ca, \\ james.zanottidadelaide.edu.au
}

\begin{abstract}
We present preliminary results on extractions of the chiral LECs $L_{10}$ and $C_{87}$ and constraints on the excited pseudoscalar state $\pi(1300)$ and $\pi(1800)$ decay constants obtained from an analysis of lattice data for the flavor $u d$ light quark V-A correlator. A comparison of the results for the correlator to the corresponding mildly-model-dependent continuum results (based primarily on experimental hadronic $\tau$ decay data) is also given.
\end{abstract}

The 30th International Symposium on Lattice Field Theory

June 24-29, 2012

Cairns, Australia

\footnotetext{
${ }^{*}$ Speaker.
} 


\section{The V-A correlator}

We focus on the difference of flavor $u d$ vector $(\mathrm{V})$ and axial vector $(\mathrm{A})$ current-current 2-point functions, $\Pi_{V / A}^{\mu v}$, and their $J=0,1$ scalar components, $\Pi_{V / A}^{(J)}$, defined in Minkowski space by

$$
\begin{gathered}
\Pi_{V / A}^{\mu v}\left(q^{2}\right) \equiv i \int d^{4} x e^{i q \cdot x}\left\langle 0\left|T\left(J_{V / A}^{\mu}(x) J_{V / A}^{\dagger v}(0)\right)\right| 0\right\rangle \\
=\left(q^{\mu} q^{v}-q^{2} g^{\mu v}\right) \Pi_{V / A}^{(1)}\left(Q^{2}\right)+q^{\mu} q^{v} \Pi_{V / A}^{(0)}\left(Q^{2}\right)
\end{gathered}
$$

where, as usual, $Q^{2}=-q^{2}$. In what follows, we denote $\Delta \Pi^{(J)} \equiv \Pi_{V}^{(J)}-\Pi_{A}^{(J)}$. The $\Pi_{V / A}^{(J)}\left(Q^{2}\right)$, for $Q^{2}>0$, also determine the corresponding Euclidean 2-point functions

$$
\left[\Pi_{V / A}^{\mu v}\left(Q^{2}\right)\right]_{E u c l}=\left(Q^{2} \delta^{\mu v}-Q^{\mu} Q^{v}\right) \Pi_{V / A}^{(1)}\left(Q^{2}\right)-Q^{\mu} Q^{v} \Pi_{V / A}^{(0)}\left(Q^{2}\right),
$$

making the $\Pi_{V / A}^{(J)}\left(Q^{2}\right)$ accessible from lattice simulations. $\Pi_{A}^{(0)}$ and $\Pi_{A}^{(1)}$ both have kinematic poles at $Q^{2}=0$ while the $J=0+1$ sum does not. Since, beyond NLO in the chiral expansion, the pole residues involve at-present-unknown NNLO LECs, we focus on $\Delta \Pi\left(Q^{2}\right) \equiv \Pi_{V}^{(0+1)}\left(Q^{2}\right)-$ $\Pi_{A}^{(0+1)}\left(Q^{2}\right)$, which satisfies an unsubtracted dispersion relation with only physical singularities. The corresponding spectral function, $\Delta \rho(s)$, consists of a $\delta$-function at $s=m_{\pi}^{2}$ and continuum beginning at $4 m_{\pi}^{2}$. The "continuum part", $\Delta \bar{\Pi}\left(Q^{2}\right)$, of $\Delta \Pi\left(Q^{2}\right)$ results from removing the $\pi$ pole: $\Delta \Pi\left(Q^{2}\right)=\Delta \bar{\Pi}\left(Q^{2}\right)-2 f_{\pi}^{2} /\left(Q^{2}+m_{\pi}^{2}\right)$. For $s<m_{\tau}^{2}, \Delta \rho(s)$ can be determined experimentally from hadronic $\tau$ decay data [1]. Public versions are available from both ALEPH [2] and OPAL [3], with a yet-to-be-corrected problem affecting the covariance matrix of the former. Beyond $s=m_{\tau}^{2}$, an alternate representation results from fitting a physically motivated model for duality violations (DVs) [5] to integrated versions of the data [4]. The OPAL data and fitted DV model provide a dispersive determination of $\Delta \Pi\left(Q^{2}\right)$ at spacelike $Q^{2}>0$, where it can also be measured on the lattice. The dispersive result is nominally quite precise, but has some (mild) model-dependence from the use of the DV model. It also involves $\tau \rightarrow 4 \pi v_{\tau}$ contributions in the $V$ channel whose uncertainties may have been underestimated, given that the corresponding $4 \pi$ branching fractions differ from expectations based on CVC and measured $e^{+} e^{-} \rightarrow 4 \pi$ cross-sections by much more than is typical for isospin-breaking corrections $[6,7]$. The mildness of the model-dependence follows from the fact that, in the range $Q^{2}<(500 \mathrm{MeV})^{2}$ expected to be of relevance to the determination of chiral LECs, DV contributions to $\Delta \rho(s)$ account for a few to several \% of $\Delta \bar{\Pi}\left(Q^{2}\right)$, the precise values depending on the point in the spectrum at which one switches from data to the fitted DV model. The cross-check on the lattice results for $\Delta \bar{\Pi}$ provided by the dispersive representation is also useful in light of the freedom to vary $m_{u, d, s}$ on the lattice, which, in principle, provides access to NNLO chiral LECs currently unknown and/or difficult to extract reliably with continuum methods.

\section{Lattice data for $\Delta \Pi\left(Q^{2}\right)$}

$\Delta \Pi\left(Q^{2}\right)$ has been determined for the fine $1 / a=2.28 \mathrm{GeV}, m_{\pi}=289,345$ and $394 \mathrm{MeV}$, and coarse $1 / a=1.37 \mathrm{GeV}, m_{\pi}=171$ and $248 \mathrm{RBC} / \mathrm{UKQCD}$ DWF ensembles detailed in Refs. [8, 9]. The latter provide an increased number of low- $Q^{2}$ points, improving the determination of the chiral 
LECs. The values of $f_{\pi}$ and $m_{\pi}$ needed to convert $\Delta \Pi\left(Q^{2}\right)$ to $\Delta \bar{\Pi}\left(Q^{2}\right)$ are given in Refs. [8, 9]. The simulation $m_{s}$ values are, in all cases, close, but not exactly equal to the physical value. As the $S U$ (2) LECs correspond to physical $m_{s}$, we analyze the data in the chiral $S U$ (3) framework. The ensemble $m_{K}$ values required for this purpose are also given in Refs. [8,9]. The spectral function, $\Delta \bar{\rho}$, of $\Delta \bar{\Pi}$, and hence also $\Delta \bar{\Pi}$ itself, are $O\left(m_{\ell}^{0}\right)$ in the chiral expansion. We thus expect the lattice $\Delta \bar{\Pi}\left(Q^{2}\right)$ to approach the physical results for sufficiently light $m_{\ell}$. We find that, within errors, for the low- $Q^{2}$ region of interest to us here, the lattice $\Delta \bar{\Pi}\left(Q^{2}\right)$ agree well with one another, and with the continuum OPAL+DV model results, for all but the $m_{\pi}=394 \mathrm{MeV}$ case, as shown in Fig. 1 .

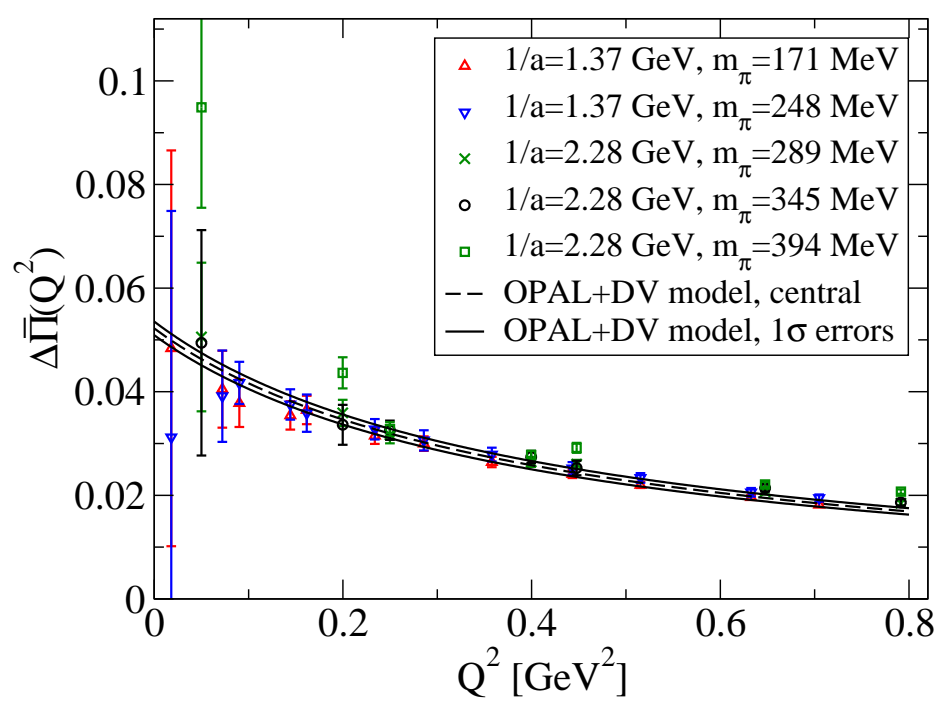

Figure 1: Lattice and OPAL+DV model results for $\Delta \bar{\Pi}\left(Q^{2}\right)$

\section{The chiral LECs}

At NLO in the chiral expansion, $\Delta \Pi$ is controlled by the single NLO LEC $L_{10}^{r}(\mu)$. Two previous lattice studies determined $L_{10}^{r}(\mu)$ by analyzing $\Delta \Pi^{(1)}[10,11]$ at NLO. With the lattice spacings available, the second-smallest non-zero $Q^{2}$ were found to be too large $\left(\sim(650 \mathrm{MeV})^{2}\right.$ and $\sim(460 \mathrm{MeV})^{2}$ for Refs. [10] and [11] respectively) to allow for a successful NLO fit. Final NLO analysis results were thus based on the single lowest $Q^{2}$ values, $(320 \mathrm{MeV})^{2}$ and $\sim(230 \mathrm{MeV})^{2}$, respectively. The current analysis improves on the previous ones in a number of ways. First, the new coarser lattices allows access to an increased number of low- $Q^{2}$ points. Second, the statistics for the $m_{\pi}=289 \mathrm{MeV}$ ensemble considered previously [11] have now been doubled. Third, because the residue of the $Q^{2}=0$ kinematic pole in $\Delta \Pi^{(1)}$ involves an unknown NNLO contribution, whose contribution, relative to that of the term involving the NLO constant $L_{10}^{r}$, gets enhanced when one 
goes to the low $Q^{2}$ desirable for extracting LECs, we switch to analyzing $\Delta \bar{\Pi}$ rather than $\Delta \Pi^{(1)}$, the $\pi$ pole contribution which must be subtracted to obtain the former having both an exactly known residue and being farther from the region of the lattice data than is the kinematic pole in $\Delta \Pi^{(1)}$. The NLO results for $L_{10}^{r}(\mu)$, for $\mu=\mu_{0}=0.77 \mathrm{GeV}$, obtained for each $Q^{2}<0.25 \mathrm{GeV}^{2}$, and all but the heaviest $m_{\pi}=394 \mathrm{MeV}$ ensemble, are shown in Fig. 2. Also shown for comparison are the results of an NLO analysis of the OPAL+DV model over a similar range of $Q^{2}$.

Since the $Q^{2}$-dependence of $\Pi_{V}\left(Q^{2}\right)$ is known to be poorly reproduced by the NLO representation [12], one might be surprised by the relative stability of the results for different $Q^{2}$. However, the missing intermediate $\rho$ contribution believed responsible for the NLO $\Pi_{V}$ slope problem [12] is encoded in the NNLO LEC $C_{93}^{r}$ [13], and the contribution to $\Pi_{V}\left(Q^{2}\right)$ proportional to $C_{93}^{r}$ is exactly cancelled by that proportional to $C_{93}^{r}$ in the NNLO expression for $\Pi_{A}^{(0+1)}\left(Q^{2}\right)$. Nonetheless, the central value for the average slope of $\Delta \bar{\Pi}\left(Q^{2}\right)$ with respect to $Q^{2}$ is significantly larger than expected from the NLO expression, albeit at only the $\sim 2 \sigma$ level. The structure of the full NNLO result, known from the results of Ref. [13], is very linear in $Q^{2}$ for the $Q^{2}$ considered here, so a significant portion of the NNLO contribution is easily removed by fitting the results of Fig. 2 to a linear form and using this to extrapolate to $Q^{2}=0$. The only NNLO contributions remaining to be removed are then those entering $\Delta \bar{\Pi}(0)$. These involve two NNLO LEC combinations, $C_{61}^{r}-C_{12}^{r}-C_{80}^{r}$, which is not large- $N_{c}$ suppressed, and $C_{62}^{r}-C_{13}^{r}-C_{81}^{r}$, which is $[13,14]$. The first combination has been estimated in Ref. [14] using the results of previous continuum works. The second is currently unknown, and has only been loosely bounded, using rough large- $N_{c}$-suppression arguments [14]. The resulting NNLO LEC combination assessments were used in Ref. [14] to obtain a continuum extraction of $L_{10}^{r}$ and $C_{87}^{r}$ (the NNLO LEC expected to dominate the slope of $\Delta \bar{\Pi}$ ). The analysis employed the ALEPH data and was based on two additional, not explicitly tested, assumptions, namely (i) that the NNLO form will successfully represent $\Delta \bar{\Pi}\left(Q^{2}\right)$ and (ii) that the V and A channel DV spectral contributions which, being governed by the resonance structure in the channel in question, are expected to be channel-dependent, can be assumed to be approximately the same in form and hence combined into a single DV ansatz for the V-A difference. While the latter assumption is not borne out by the combined V and A channel fits of Refs. [4], the contribution to $\Delta \bar{\Pi}$ of the DV part of $\Delta \bar{\rho}$ is small in the low- $Q^{2}$ region. It is thus of interest to compare the results of our fit to those of this mildly model-dependent continuum analysis, which are [14]

$$
L_{10}^{r}\left(\mu_{0}\right)=-0.0041(4)_{N N L O} ; \quad C_{87}^{r}\left(\mu_{0}\right)=0.0049(2)_{N N L O} \mathrm{GeV}^{-2},
$$

with the error dominated entirely by the uncertainty in the estimate for unknown large- $N_{c}$-suppressed NNLO LEC combination. Results obtained using instead the OPAL data, and incorporating the results of the DV model fits of Refs. [4] in order to remove the second of the two assumptions noted above, are in extremely close agreement. An additional unknown systematic error, associated with the two additional assumptions noted above, of course also exists for the results of Eq. (3.1). Our final goal is to perform a NNLO analysis of the lattice data, including a range of $m_{q}$ sufficient to put constraints on the currently unknown NNLO LECs (something likely to be feasible given the difference of the results for $\Delta \bar{\Pi}$ for $m_{\pi}=394 \mathrm{MeV}$ from those for the smaller $m_{\pi}$ seen in Fig. 1), but this analysis has not yet been completed. The results of following the continuum estimates/arguments 
of Ref. [14] for the unknown NNLO LEC combinations entering $\Delta \bar{\Pi}(0)$ are

$$
L_{10}^{r}\left(\mu_{0}\right)=-0.0038(4)_{l a t t}(4)_{N N L O} ; \quad C_{87}^{r}\left(\mu_{0}\right)=0.0040(21)_{\text {latt }}(2)_{N N L O} \mathrm{GeV}^{-2},
$$

in good agreement with, though less precise than the NNLO-LEC-induced part of the error obtained explicitly in the continuum analysis of Ref. [14].

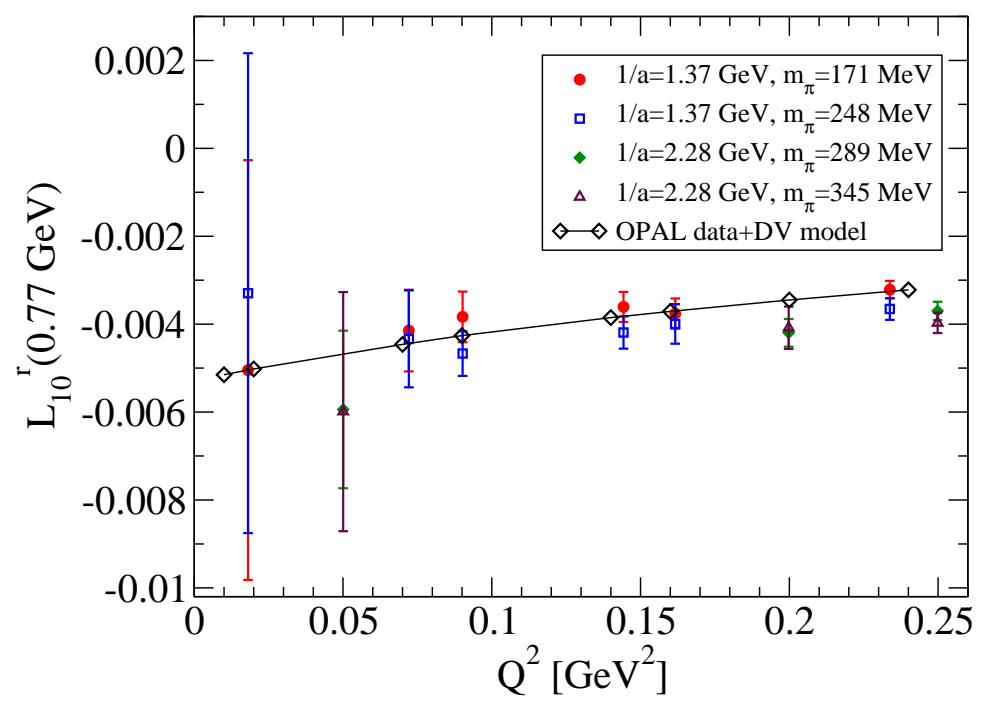

Figure 2: NLO results for $L_{10}^{r}\left(\mu_{0}\right)$ from analyses of lattice and OPAL+DV model versions of $\Delta \bar{\Pi}$

\section{Constraints on the $\pi^{\prime}$ and $\pi^{\prime \prime}$ Decay Constants}

Excited $I=1$ pseudoscalar mesons, $P$, couple with strengths $2 f_{P} m_{P}^{2}$ to the divergence of the flavor $u d$ axial current. Their decay constants, $f_{P}$, enter the conventional determination of $m_{u}+m_{d}$ employing sum rules for the two-point function of this divergence [16] and are currently determined as part of the analysis. A similar internal determination is required in the extraction of $m_{u}+m_{s}$ from sum rules for the two-point function of the divergence of the flavor us axial current. Finally, flavorbreaking sum rules used to determine $\left|V_{u s}\right|$ from hadronic $\tau$ decay $[17,18]$, or a combination of hadronic $\tau$ decay and electroproduction cross-section data [19], encounter a problem with the very bad convergence of the OPE representation of $J=0$ contributions, necessitating the subtraction of the chirally suppressed, but not totally negligible, strange excited state scalar and pseudoscalar contributions to the differential $\tau$ decay spectrum. The strange pseudoscalar subtraction relies on the excited $K$ decay constants obtained in the sum rule analysis.

The lattice data allows us to test the reliability of the sum rule determination of such decay constants, as follows. The quantity $P\left(Q^{2}\right) \equiv Q^{2} \Delta \Pi^{(0)}\left(Q^{2}\right)$, which, for $m_{u}=m_{d}$ is equal to 
$-Q^{2} \Pi_{A}^{(0)}\left(Q^{2}\right)$, is free of kinematic singularities and satisfies a once-subtracted dispersion relation. Since $\Pi_{A}^{(0)}\left(Q^{2}\right)$ and the quantities $m_{\pi}, f_{\pi}$ which determine the pion pole contribution to the dispersive representation are all measurable on the lattice, the following rearranged version of this relation provides constraints on the continuum contribution, and hence on the excited state decay constants, $f_{\pi^{\prime}}$ and $f_{\pi^{\prime \prime}}$, for each pair of $Q^{2}$ and subtraction point $Q_{0}^{2}$ :

$$
P\left(Q^{2}\right)-P\left(Q_{0}^{2}\right)+\frac{\left(Q^{2}-Q_{0}^{2}\right) 2 f_{\pi}^{2} m_{\pi}^{2}}{\left(s+Q^{2}\right)\left(s+Q_{0}^{2}\right)}=-\left(Q^{2}-Q_{0}^{2}\right) \int_{9 m_{\pi}^{2}}^{\infty} d s \frac{s \rho_{A}^{(0)}(s)}{\left(s+Q^{2}\right)\left(s+Q_{0}^{2}\right)} .
$$

Spectral positivity ensures that the LHS provides an upper bound on the contributions from any subset of the full set of excited pseudoscalar states. In the narrow width approximation, this constraint represents a straight line in the $f_{\pi^{\prime}}^{2} f_{\pi^{\prime \prime}}^{2}$ plane for each pair $\left(Q^{2}, Q_{0}^{2}\right)$. The fact that the excited state decay constants scale as $m_{\pi}^{2}$ can be used to scale each such bound from the masses used in the simulation down to physical $m_{\pi}$. It turns out that, at present, only the high-statistics, $1 / a=2.28 \mathrm{GeV}$, $m_{\pi}=289 \mathrm{MeV}$ ensemble provides data sufficiently accurate for this purpose. The envelope of the resulting set of constraint lines, scaled down to physical $m_{\pi}$, is shown in Fig. 3. Also shown, for comparison, are the results obtained/used in Refs. [16]. These are obviously in good agreement with the lattice constraints, leaving room for small additional contributions to the dispersive representation from yet higher excited pseudoscalar resonances.

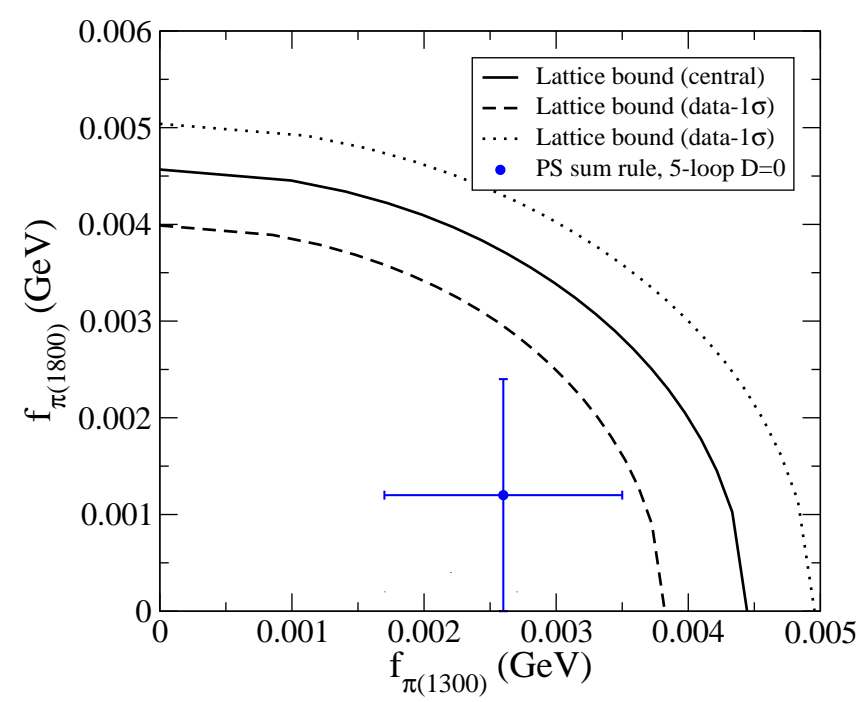

Figure 3: Lattice constraints on the $\pi^{\prime}$ and $\pi^{\prime \prime}$ decay constants

\section{Acknowledgements}

The computations were done using the STFC's DiRAC facilities at Swansea and Edinburgh. PAB, LDD, NG and RJH are supported by an STFC Consolidated Grant, and by the EU under 
Grant Agreement PITN-GA-2009-238353 (ITN STRONGnet). EK was supported by the Comunidad Autònoma de Madrid under the program HEPHACOS S2009/ESP-1473 and the European Union under Grant Agreement PITN-GA-2009-238353 (ITN STRONGnet). KM acknowledges the hospitality of the CSSM, University of Adelaide, and support of NSERC (Canada). JMZ is supported by the Australian Research Council grant FT100100005.

\section{References}

[1] Y.-S. Tsai, Phys. Rev. D4 (1971) 2821; E. Braaten, S. Narison, A. Pich, Nucl. Phys. B373 (1992) 581.

[2] S. Schael et al. [ALEPH Collaboration], Phys. Rep. 421 (2005) 191 [hep-ex/0506072].

[3] K. Ackerstaff et al. [OPAL Collaboration], Eur. Phys. J. C7 (1999) 571 [hep-ex/9808019].

[4] D. Boito, it et al., Phys. Rev. D84 (2011) 113006 [arXiv: 1110.1127 (hep-ph) ]; ibid. D85 (2012) 093015 [arXiv:1203.3146 (hep-ph)].

[5] See O. Catà, M. Golterman and S. Peris, Phys. Rev. D77 (2008) 093006 [arXiv : 0803.0246 (hep-ph) ] and earlier references cited therein.

[6] M. Davier, S. Eidelman, A. Hocker, Z. Zhang, Eur. Phys. J. C27 (2003) 497 [hep-ph/ 0208177 ].

[7] See, e.g., the $\tau 2012$ talk of B. Schwartz for more on the $e^{+} e^{-} \rightarrow \pi^{+} \pi^{-} \pi^{0} \pi^{0}$ cross-sections, where the bulk of the discrepancy remains between $\tau$ and $\mathrm{CVC}+$ electroproduction expectations.

[8] Y. Aoki et al., Phys. Rev. D83 (2011) 074508 [arXiv:1011.0892 (hep-lat)].

[9] R. Arthur et al., arXiv:1208.4412 (hep-lat).

[10] E. Shintani, et al., Phys. Rev. Lett. 101 (2008) 202004 [arXiv: 0806.4222 (hep-lat) ].

[11] P.A. Boyle, L. Del Debbio, J. Wennekers and J.M. Zanotti, Phys. Rev. D81 (2010) 014504 [arXiv:0909.4931 (hep-lat)].

[12] C. Aubin and T. Blum, Phys. Rev. D75 (2006) 114502 [hep-lat/ 0608011$].$

[13] G. Amoros, J. Bijnens and P. Talavera, Nucl. Phys. B568 (2000) 319 [hep-ph / 9907264$]$

[14] M. Gonzalez-Alonso, A. Pich, J. Prades, Phys. Rev. D78 (2008) 116012 [arXiv: 0810 . 0760 (hep-ph) ].

[15] K. Maltman Phys. Rev. D58 (1998) 093015 [hep-ph/ 9804298 ]; K. Maltman and J. Kambor, ibid. D64 (2001) 093014 [hep-ph/ 0107187$].$

[16] See, e.g., K. Maltman and J. Kambor, Phys. Rev. D65 (2002) 074013 [hep-ph / 0108227$]$; K.G. Chetyrkin and A. Khodjamirian, Eur. Phys. J. C46 (2006) 721 [hep-ph/ 0512295 ].

[17] E. Gamiz, et al., JHEP 0301 (2003) 060 [hep-ph / 0212230$]$; Phys. Rev. Lett. 94 (2005) 011803 [hep-ph/0408044].

[18] K. Maltman and C.E. Wolfe, Phys. Lett. B639 (2006) 283 [hep-ph / 0603215$]$; K. Maltman, et al., Nucl. Phys. Proc. Suppl. 189 (2009) 175 [arXiv: 0906.1386$].$

[19] K. Maltman, Phys. Lett. B672 (2009) 257 [arXiv: 0811 . 1590]. 